\title{
PaleoEcoGen: Unlocking the power of ancient environmental DNA to understand past ecological trends
}

\author{
Marie-Eve Monchamp1,2, L. Armbrechtt, , E. Capo ${ }^{5}$, M.J.L. Coolen6, T. Cordier', I. Domaizon ${ }^{8}$, L.S. Epp , \\ C. Giguet-Covex ${ }^{10}$, I. Gregory-Eaves ${ }^{1,2}$, U. Herzschuh ${ }^{11}$, L. Parducci' ${ }^{12}$, K.R. Stoof-Leichsenring ${ }^{11}$ and J.W. Williams ${ }^{13}$
}

PaleoEcoGen is a new working group that was launched with the aim of bringing together scientists from around the world who use ancient environmental DNA (ancient eDNA) as a novel proxy to examine the response of past biological communities to environmental changes (pastglobalchanges. org/paleoecogen). We are particularly interested in exploiting the added value of these emerging ancient eDNA tools to advance our knowledge of critical transitions in Earth's Quaternary history. To this end, we aim to stimulate and enhance international ancient eDNA research by organizing topical workshops to discuss new methodologies in the field (including synthetic analyses and modeling approaches), and to coordinate research efforts for bigger picture analyses that, ultimately, will help to inform conservation efforts and future biodiversity assessments.

Changes in ecosystem dynamics can occur gradually over centuries to millennia, or abruptly (i.e. at decadal to annual timescales). Rapid changes may challenge the fitness and survival of organisms, including those that are essential for ecosystem maintenance. Even small disturbances may weaken the stability and resilience of an ecosystem (Fig. 1), and ultimately lead to "critical transitions" where the system is pushed from one equilibrium state to another (Taranu et al. 2018). These "tipping points" are often hard to predict because of the complexity of the interactions between organisms and their environment, and they imply prolonged ecosystem consequences that may not be reversible.

Critical transitions have been documented for terrestrial and aquatic ecosystems, as well as social-ecological systems, and they have been studied across many scientific disciplines (Scheffer et al. 2009; 2012). In the context of global changes, especially the "Great Acceleration" (Steffen et al. 2015), studying critical transitions has been identified as a priority in paleoecological research by the scientific community (Seddon et al. 2014). With new methodological approaches on the rise in the paleosciences, we now have the opportunity to describe past critical transitions and their effects on biological communities (Taranu et al. 2018; Capo et al. 2021).

Our working group is motivated to address two key questions:

- How can we use (sedimentary) ancient eDNA timeseries to better identify and characterize past critical transitions?

-What are the subsequent evolutionary and ecological trajectories, and which projections for future biodiversity and ecosystem change can be drawn from past critical transitions during the Quaternary?

The detailed study of critical transitions in paleoecology requires the generation of the most comprehensive view possible-of an ecosystem, its drivers, and their interactions. To meet this challenge, stratigraphic analysis of ancient eDNA is a key analytical approach because of its potential to provide new insights into: (1) the composition of biological communities across multiple trophic levels including organisms that do not fossilize; (2) species interactions within communities; and (3) the response of organisms, from individual taxa to communities, to past environmental changes (Coolen et al. 2013; Domaizon et al. 2017; Schulte et al. 2021; Liu et al. 2021). Like any other proxy, ancient eDNA has its limitations (Capo et al. 2021), but the field is now sufficiently mature to offer exciting new opportunities to expand our knowledge using paleoenvironmental data.

\section{Upcoming activities}

Our first online workshop will be in 2022 in collaboration with the sedaDNA scientific society (ercapo.wixsite.com/sedadna-society). The workshop will be dedicated to improving inclusion of African ancient eDNA researchers by offering a collaborative platform and training opportunities in molecular

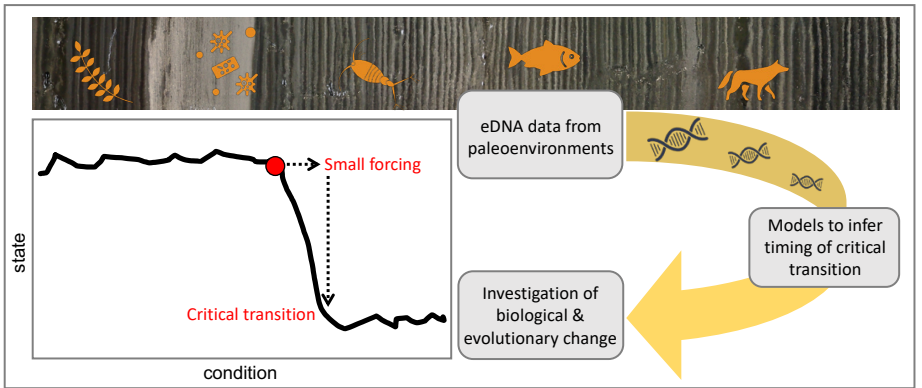

Figure 1: (Left) Schematic representation of a critical transition between two states triggered by a small forcing (Right) Simplified workflow of the proposed approach to identify past critical transitions and evaluate subsequent biological changes based on ancient environmental DNA timeseries. techniques applied to sedimentary ancient eDNA. A second workshop (in-person or online, depending on COVID-19 pandemic regulations) will focus on developing a multivariate modeling approach based on ancient eDNA temporal data (Taranu et al. 2018) to investigate the timing and magnitude of shifts in paleoenvironmental records.

Visit our website (pastglobalchanges.org/ paleoecogen) and register to our mailing list to keep up to date with our activities and find out how to get involved. PaleoEcoGen is also on Twitter: @PaleoEcoGen

\section{AFFILIATIONS}

'Department of Biology, McGill University, Montreal, Canada

${ }^{2}$ Groupe de recherche interuniversitaire en limnologie (GRIL), Montreal, Canada

${ }^{3}$ Australian Centre for Ancient DNA, School of

Biological Sciences, The University of Adelaide, Australia

4nstitute for Marine and Antarctic Studies, Centre for Ecology and Biodiversity, Tasmania, Australia ${ }^{5}$ Department of Aquatic Sciences and Assessment, Swedish University of Agricultural Sciences, Uppsala, Sweden

Western Australia Organic and Isotope Geochemistry Centre (WA-OIGC), School of Earth and Planetary Sciences, Curtin University, Bentley, Australia 'NORCE Climate, NORCE Norwegian Research Centre AS, Bjerknes Centre for Climate Research, Bergen, Norway

${ }^{8}$ INRAE, CARRTEL, USMB, Thonon les bains, France 'Limnological Institute, Department of Biology, University of Konstanz, Germany

${ }^{\circ}$ Environnements Dynamiques et Territoires de la Montagne, Université Savoie Mont Blanc, Le Bourget du Lac, France

"Alfred Wegener Institute Helmholtz Centre for Polar and Marine Research, Potsdam, Germany

${ }^{12}$ Department of Environmental Biology, La Sapienza University of Rome, Italy

${ }^{13}$ Department of Geography, University of Wisconsin, Madison, WI, USA

\section{CONTACT}

Marie-Eve Monchamp: me.monchamp@gmail.com

\section{REFERENCES}

\section{Capo E et al. (2021) Quaternary 4: 1-58}

Coolen MJL et al. (2013) Proc Natl Acad Sci USA 110: 8609-8614

Domaizon I et al. (2017) J Paleolimnol 58: 1-21

Liu S et al. (2021) Nat Commun 12: 2995 More KD et al. (2018) Earth Planet Sci Lett 496: 248-256 Scheffer M et al. (2009) Nature 461: 53-59 Scheffer M et al. (2012) Science 338: 344-348 Schulte L et al. (2021) Mol Ecol Resour 21: 801-815 Seddon AWR et al. (2014) J Ecol 102: 256-267 Steffen W et al. (2015) Science 347:1259855 Taranu ZE et al. (2018) Ecosphere 9: 1-18 